\title{
Improvement of Neuroenergetics by Hypertonic Lactate Therapy in Patients with Traumatic Brain Injury Is Dependent on Baseline Cerebral Lactate/Pyruvate Ratio
}

\author{
Hervé Quintard, ${ }^{1,2, *}$ Camille Patet,, ${ }^{1, *}$ Jean-Baptiste Zerlauth, ${ }^{3}$ Tamarah Suys, ${ }^{1}$ Pierre Bouzat, ${ }^{1,4}$ Luc Pellerin, ${ }^{5}$ \\ Reto Meuli, ${ }^{3}$ Pierre J. Magistretti, ${ }^{6-8}$ and Mauro Oddo ${ }^{1}$
}

\begin{abstract}
Energy dysfunction is associated with worse prognosis after traumatic brain injury (TBI). Recent data suggest that hypertonic sodium lactate infusion (HL) improves energy metabolism after TBI. Here, we specifically examined whether the efficacy of $\mathrm{HL}$ ( $3 \mathrm{~h}$ infusion, $30-40 \mu \mathrm{mol} / \mathrm{kg} / \mathrm{min}$ ) in improving brain energetics (using cerebral microdialysis [CMD] glucose as a main therapeutic end-point) was dependent on baseline cerebral metabolic state (assessed by CMD lactate/pyruvate ratio [LPR]) and cerebral blood flow (CBF, measured with perfusion computed tomography [PCT]). Using a prospective cohort of 24 severe TBI patients, we found CMD glucose increase during HL was significant only in the subgroup of patients with elevated CMD LPR $>25(n=13 ;+0.13$ [95\% confidence interval (CI) $0.08-0.19] \mathrm{mmol} / \mathrm{L}, p<0.001$; vs. $+0.04[-0.05-0.13]$ in those with normal LPR, $p=0.33$, mixed-effects model). In contrast, CMD glucose increase was independent from baseline CBF (coefficient $+0.13[0.04-0.21] \mathrm{mmol} / \mathrm{L}$ when global CBF was $<32.5 \mathrm{~mL} / 100 \mathrm{~g} / \mathrm{min}$ vs. $+0.09[0.04-0.14] \mathrm{mmol} / \mathrm{L}$ at normal CBF, both $p<0.005$ ) and systemic glucose. Our data suggest that improvement of brain energetics upon HL seems predominantly dependent on baseline cerebral metabolic state and support the concept that CMD LPR - rather than CBF - could be used as a diagnostic indication for systemic lactate supplementation following TBI.
\end{abstract}

Key words: cerebral blood flow; cerebral microdialysis; hypertonic; lactate; traumatic brain injury

\section{Introduction}

C ERebral energy dysfunction has recently emerged as an important determinant of prognosis following traumatic brain injury (TBI). ${ }^{1}$ Although the exact mechanisms are still not completely understood, clinical investigation using the intra-cerebral microdialysis (CMD) technique has identified reduced cerebral extracellular glucose as a surrogate marker of post-TBI cerebral energy dysfunction and outcome. ${ }^{2}$ Glucose is an essential substrate for the brain and is also important for several pathways, which are crucial for brain cell survival, for example, the pentose-phosphate pathway. ${ }^{3}$ Strategies to improve cerebral glucose therefore appear of potential benefit in this setting.

Abundant experimental evidence has demonstrated that lactate can function as an energy substrate for the brain, particularly in conditions of increased energy demand, such as after TBI. ${ }^{4,5}$ Preliminary clinical interventional trials using hypertonic sodium lactate infusion (HL) in patients with TBI have shown HL can be successfully used to reduce secondary intracranial hypertension ${ }^{6}$ or as a valid alternative to mannitol for the treatment of elevated

\footnotetext{
${ }^{1}$ Department of Intensive Care Medicine, Neuroscience Critical Care Research Group, ${ }^{3}$ Department of Medical Radiology, ${ }^{7}$ Centre de Neurosciences Psychiatriques, Department of Psychiatry, Centre Hospitalier Universitaire Vaudois (CHUV), Lausanne University Hospital, Lausanne, Switzerland.

${ }^{2}$ Department of Anesthesia and Intensive Care, Nice University Hospital, Nice, France.

${ }^{4}$ Department of Anesthesia and Intensive Care, Grenoble University Hospital, Grenoble, France.

${ }^{5}$ Institute of Physiology, University of Lausanne, Lausanne, Switzerland.

${ }^{6}$ Division of Biological and Environmental Sciences and Engineering, King Abdullah University of Science and Technology (KAUST), Thuwal, Kingdom of Saudi Arabia.

${ }^{8}$ Laboratory of Neuroenergetics and Cellular Dynamics, Brain Mind Institute, Ecole Polytechnique Fédérale de Lausanne (EPFL), Lausanne, Switzerland.

*The first two authors contributed equally.

(c) Hervé Quintard, et al., 2015; Published by Mary Ann Liebert, Inc. This Open Access article is distributed under the terms of the Creative Commons Attribution Noncommercial License (http://creativecommons.org/licenses/by-nc/4.0/) which permits any noncommercial use, distribution, and reproduction in any medium, provided the original author(s) and the source are credited.
} 
intracranial pressure (ICP). ${ }^{7}$ Using CMD monitoring, we have recently shown that HL was effective in decreasing ICP and had positive effects on cerebral energetics. ${ }^{8}$ Control of ICP and cerebral glucose-sparing effects of HL provide the pathophysiological rationale for its potential utility in the treatment of acute brain injury.

Although HL may be a valid therapeutic intervention, the exact conditions and potential indications for its utilization are still to be better characterized. The CMD lactate/pyruvate ratio (LPR) is a marker of tissue cerebral metabolic state. Elevated LPR $>25$ is a marker of impaired cerebral oxidative metabolism and outcome after TBI. ${ }^{9,10}$ The CMD LPR would therefore be an ideal marker to identify subjects with high LPR who are more likely to benefit from therapies aiming to improve neuroenergetics, such as HL. On the other extent, the majority of animal and human data on $\mathrm{HL}$ is predominantly focused on TBI, ${ }^{11}$ and only a few tested models of cerebral ischemia. ${ }^{12,13}$ Whether HL retains its efficacy in conditions of reduced cerebral blood flow (CBF) has not been evaluated so far in humans.

The specific end-point of this prospective study was therefore to examine in patients with severe TBI monitored with CMD the effect of HL on cerebral energetics - using CMD glucose as an end-point for treatment efficacy - according to baseline CMD LPR and CBF, quantified by perfusion computed tomography CT (PCT). ${ }^{14,15}$

\section{Methods}

\section{Patients}

This was a prospective interventional study performed between March 2012 and October 2014 on consecutive patients with severe TBI admitted to the Department of Intensive Care Medicine, Lausanne University Hospital, Lausanne, Switzerland. Subjects had severe TBI (defined by a Glasgow Coma Scale score $<9$ ), an abnormal head CT scan (defined by a Marshall score $\geq 2$ ), and underwent intracranial monitoring - consisting of CMD and ICP as part of standard care. The present investigation specifically focused on the relationship between the sparing-glucose effect of HL and patient CMD LPR and CBF: a subset of the cohort presented here $(15 / 24$ patients $)$ was described in our previous study. ${ }^{8}$ Approval for the study was obtained from the local Ethical Committee, and informed consent was obtained from each patient's next of kin and from an independent physician.

\section{Systemic monitoring}

Patients were treated according to a written institutional algorithm for the treatment of severe TBI, based on international guidelines and as previously described by our group. ${ }^{16}$ Sedationanalgesia consisted of propofol and sufentanil. Arterial blood concentrations of glucose and lactate were collected hourly, simultaneously to CMD samples, using an intra-arterial catheter.

\section{Cerebral microdialysis monitoring}

Cerebral metabolic monitoring was performed using an intraparenchymal CMA 70 catheter with a $20 \mathrm{kDa}$ cut-off, perfused with artificial cerebrospinal fluid via a CMA 106 pump at a rate of $0.3 \mu \mathrm{L} / \mathrm{min}$ (CMA Microdialysis AB, Stockholm, Sweden). Brain extracellular samples were collected every $60 \mathrm{~min}$ and immediately analyzed at the bedside for concentrations of glucose, lactate, and pyruvate, using a kinetic enzymatic analyzer (ISCUS ${ }^{\text {flex }}$; CMA Microdialysis AB). The CMD catheter was inserted in the operating room by an experienced neurosurgeon through a multiple-lumen bolt (Integra Neurosciences, Plainsboro, NJ) and was placed in the right frontal lobe, in apparently normal sub-cortical white matter. Adjacent to the CMD catheter, a Codman ${ }^{\circledR}$ ICP monitor (Codman, Raynham, MA) was inserted for continuous measurement of ICP. The appropriate location of all monitors was assessed by a follow-up head CT scan, performed within the first $24 \mathrm{~h}$ from intensive care unit (ICU) admission.

\section{Perfusion CT}

Perfusion acquisitions were realized during the first control head CT scan, using a LightSpeed multi-detector row CT system (GE Medical Systems, Milwaukee, WI). Scanning was initiated $5 \mathrm{sec}$ after injection of $50 \mathrm{~mL}$ of iohexol $(300 \mathrm{mg} / \mathrm{mL}$ of iodine; GE Healthcare Europe, Glattbrugg, Switzerland), perfused at a rate of $5 \mathrm{~mL} / \mathrm{sec}$ with the following parameters: $80 \mathrm{kV}, 240 \mathrm{mAs}, 0.4$ rotations/sec, total duration of $50 \mathrm{sec}$. The series evaluated 16 adjacent 5-mm-thick sections of brain parenchyma. A dedicated software (Brilliance Workspace Portal ${ }^{\circledR}$; Philips Medical Systems, Cleveland, $\mathrm{OH}$ ), which employs the central volume principle using deconvolution to measure mean transit time (MTT), was used. Cerebral blood volume (CBV) was calculated from the timeenhancement curves, and global $\mathrm{CBF}$ was derived from the equation: $\mathrm{CBF}=\mathrm{CBV} / \mathrm{MTT}$. Three-dimensional reconstruction was processed with Carestream Vue PACS ${ }^{\circledR}$ (Carestream Health, Rochester, NY) using a series of the thin-slice enhanced brain CT. Regions of interest (ROI) were selected in line with our previous studies providing an accurate quantitative assessment of CBV, MTT, and CBF of each hemisphere. ${ }^{14,15}$ A global value of CBV, MTT, and CBF was calculated by averaging values from right and left hemispheres. Post-processing of PCT data was performed by an experienced neuroradiologist who was blinded to $\mathrm{CMD}$ variables.

\section{Study intervention}

Intervention consisted of a continuous infusion of hypertonic sodium lactate $\left(\mathrm{Na}^{+} 1000 \mathrm{mmol} / \mathrm{L}\right.$ and lactate $1000 \mathrm{mmol} / \mathrm{L}$, prepared by the Division of Pharmacy, CHUV, Lausanne, Switzerland), administered over $3 \mathrm{~h}$, at a concentration of $30-40 \mu \mathrm{mol} / \mathrm{kg} / \mathrm{min}$, initiated as soon as possible following PCT. Dosing and timing of the therapy were based on previous clinical trials conducted on healthy subjects ${ }^{17-19}$ and critically ill patients, ${ }^{20}$ aiming to reach a steady concentration of arterial blood lactate of about $4-5 \mathrm{mmol} / \mathrm{L}$.

\section{Data collection and processing}

Patient characteristics and radiological variables included age, admission Glasgow Coma Scale score, Marshall CT-scan score, time from TBI to CMD monitoring and to the start of HL, time from PCT to the start of HL, and duration of intracranial monitoring.

Patients were categorized into two subgroups according to a) baseline CMD LPR, using LPR $>25$ as the threshold of impaired cerebral oxidative metabolism and increased energy demand, in line

Table 1. Patient Baseline Characteristics

\begin{tabular}{lc} 
Variable & Value \\
\hline Patient number & 24 \\
Age, years & $38[24-54]$ \\
Cause of injury, fall/RTC & $8 / 16$ \\
Isolated TBI vs. polytrauma & $17 \mathrm{vs.7}$ \\
Decompressive craniectomy, yes/no & $2 / 22$ \\
Admission Glasgow Coma Scale score & $6[5-8]$ \\
Marshall CT-score & $2[2-3]$ \\
Time from TBI to initiation of CMD monitoring, h & $8[6-16]$ \\
Time from TBI to initiation of HL, h & $27[22-41]$ \\
Time from PCT to initiation of HL, h & $4[2-18]$ \\
Duration of CMD monitoring, days & $4[3-7]$
\end{tabular}

Data are expressed as median and interquartile range.

CMD, cerebral microdialysis; HL, hypertonic lactate infusion; PCT, perfusion CT; RTC, road traffic collision; TBI, traumatic brain injury. 
Table 2. Baseline Cerebral and Systemic Physiological Variables

\begin{tabular}{lc}
\hline Variable & Value \\
\hline CMD glucose, $\mathrm{mmol} / \mathrm{L}$ & $1.5[0.5-4.3]$ \\
CMD lactate, $\mathrm{mmol} / \mathrm{L}$ & $2.8[1.2-4.1]$ \\
$\mathrm{CMD} \mathrm{LPR}$ & $24[13-45]$ \\
$\mathrm{CBF}, \mathrm{mL} / 100 \mathrm{~g} / \mathrm{min}$ & $49.8[24.7-82.8]$ \\
Arterial blood glucose, $\mathrm{mmol} / \mathrm{L}$ & $7.4[5.3-10.7]$ \\
Arterial blood lactate, $\mathrm{mmol} / \mathrm{L}$ & $1[0.6-4.3]$ \\
\hline
\end{tabular}

Data are expressed as median and ranges.

CBF, cerebral blood flow; CMD, cerebral microdialysis; LPR, lactate/ pyruvate ratio.

with previous studies ${ }^{9,10}$; and b) baseline global CBF, dichotomized as normal versus. oligemic, defined by a $\mathrm{CBF}<32.5 \mathrm{~mL} / 100 \mathrm{~g} / \mathrm{min}$. This cut-off for global CBF was based on our previous PCT studies in TBI patients and was set to be at least 2 standard deviations below normal $\mathrm{CBF}^{14,15}$

CMD samples were analyzed hourly, simultaneously to arterial blood gas analysis. Baseline CMD values were defined as the single value taken from the sample immediately preceding the start of HL. Systemic values were recorded continuously and were then matched to timely CMD samples.

\section{Statistical analysis}

Dynamic hourly changes of arterial blood and CMD glucose and lactate concentrations from baseline (T0), during HL (T0-3 h), and up to $12 \mathrm{~h}$ were first graphically plotted. For comparisons, longitudinal data analysis was used to account for repeated measures of physiological variables over time across different patients. Statistical analysis was conducted with a mixed-effects multi-level regression model, with time entered as an independent variable and allowing it to have a random intercept and a random slope according to the patient. A correlation coefficient (with 95\% CI) was obtained, which corresponded to the change of the variable during the time of HL. This analysis was conducted in the total population and in predefined sub-groups. Statistical analyses were performed using Stata 12 (Stata Corp., College Station, TX), and statistical significance was set at $p<0.05$.

\section{Results}

\section{Patient characteristics}

A total of 24 consecutive patients were studied. Baseline demographic and physiological characteristics are presented in Table 1 and Table 2, respectively.

\section{Dynamic changes of arterial blood and CMD glucose and lactate levels during $H \mathrm{~L}$}

Figure 1 illustrates changes of CMD (A) and arterial blood (B) concentrations of lactate and glucose over time. Table 3 summarizes the results of a mixed-effects regression model, showing that HL was associated with a significant increase in the concentrations of CMD glucose, CMD lactate, and arterial blood lactate.

\section{Cerebral glucose-sparing effect of $H L$ was independent from systemic glucose}

Systemic lactate can be converted into glucose via hepatic gluconeogenesis (GNG), which is activated in subjects with $\mathrm{TBI}^{21}$; therefore, the observed increase in CMD glucose upon HL could be at least partly secondary to systemic glucose increase via GNG, which is subsequently transported to the brain. We found that although arterial blood glucose increased slightly during HL, this was not statistically significant ( $p=0.16$; Table 3 ), thereby indicating HL-related increase of cerebral extracellular glucose was not dependent from arterial blood glucose, but rather predominantly attributable to a cerebral sparing-glucose effect. Importantly, systemic glucose always remained within the ranges for clinical safety after TBI $(6-10 \mathrm{mmol} / \mathrm{L})$, and it was not accompanied by a significant change in intravenous insulin dose (Table 3 ).

\section{Effect of HL on cerebral glucose according to baseline brain $L P R$ and $C B F$}

The effect of HL on cerebral energetics was then examined in predefined subgroups, according to patients' baseline CMD LPR and $\mathrm{CBF}$, focusing this subgroup analysis on the main therapeutic endpoint, that is, CMD glucose. Figure 2 shows the dynamic changes of CMD glucose over time, as a function of LPR (A) and CBF (B).

Mixed-effects model statistical analysis is summarized in Table 4. When dichotomizing TBI subjects by baseline LPR, CMD glucose increased significantly only in the subgroup of patients with baseline elevated LPR $>25(n=13$; correlation coefficient $+0.13 \mathrm{mmol} / \mathrm{L}$ [95\% CI 0.08-0.19] during HL, $p<0.0001$ ), whereas no significant increase in CMD glucose was observed in patients with normal LPR $(n=11$; Table 4).

In contrast, $\mathrm{CMD}$ glucose increase following $\mathrm{HL}$ was comparable in patients with oligemic $\mathrm{CBF}(n=8$; average CBF 29.6 [range 24.732] $\mathrm{mL} / 100 \mathrm{~g} / \mathrm{min}$; correlation coefficient +0.13 [95\% CI $0.04-0.21$ ] $\mathrm{mmol} / \mathrm{L}$ during $\mathrm{HL}, p=0.003)$ and normal CBF $(n=14$; average CBF 55.0 [41.6-82.8] $\mathrm{mL} / 100 \mathrm{~g} / \mathrm{min}$; correlation coefficient $+0.09[95 \%$ CI 0.04-0.14] mmol/L during HL, $p=0.001$ ).
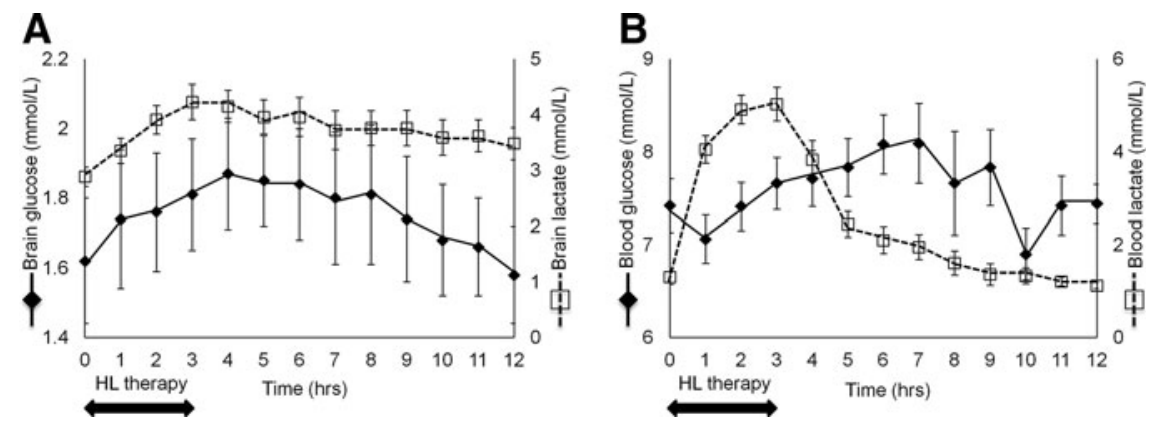

FIG. 1. Dynamic changes of blood and cerebral extracellular levels of glucose and lactate from baseline, during and following hypertonic lactate infusion (HL). The graph illustrates hourly brain extracellular (A) and arterial blood (B) concentrations of glucose (black lines) and lactate (dashed lines) from baseline up to $12 \mathrm{~h}$ following the start of 3-h intravenous HL. Data are expressed as mean \pm standard error of mean. 
Table 3. Changes in Cerebral Extracellular and Arterial Blood Concentrations of Glucose and Lactate during Hypertonic Lactate Infusion

\begin{tabular}{lccc} 
Variable & Coefficient \pm SE & 95\% CI & P value \\
\hline Cerebral glucose, mmol/L & $0.08 \pm 0.02$ & $0.03-0.12$ & 0.001 \\
Cerebral lactate, mmol/L & $0.35 \pm 0.07$ & $0.22-0.48$ & $<0.0001$ \\
Systemic glucose, $\mathrm{mmol} / \mathrm{L}$ & $0.12 \pm 0.08$ & $-0.045-0.28$ & 0.16 \\
Insulin dose, units/h & $0.05 \pm 0.19$ & $-0.32-0.42$ & 0.26 \\
Systemic lactate, $\mathrm{mmol} / \mathrm{L}$ & $0.44 \pm 0.10$ & $0.24-0.63$ & $<0.0001$ \\
\hline
\end{tabular}

$P$ values were calculated with a mixed-effects multilevel regression model, to account for repeated measures across different patients over time.

CI, confidence interval; SE, standard error.

\section{Discussion}

The main findings of this study can be summarized as follows: 1) HL improves cerebral energetics following TBI, independent of systemic glucose; 2) cerebral glucose-sparing effect by HL is significant only in patients with impaired cerebral oxidative metabolism, as defined by CMD LPR >25; and 3) cerebral glucose increase seems not to differ in patients with normal versus oligemic $\mathrm{CBF}<32.5 \mathrm{~mL} / 100 \mathrm{~g} / \mathrm{min}$.

\section{Energy dysfunction after TBI}

Glucose is the main energy substrate for the brain. ${ }^{3}$ Glucose crosses the blood-brain barrier via specific glucose transporters (GLUT), proportionally to baseline metabolism and demand. ${ }^{22}$ After TBI, energy demand is considerably augmented, causing an increase in cerebral metabolic rate of glucose and so-called hyperglycolysis. ${ }^{23}$ This may ultimately lead to energy dysfunction or crisis, with concomitant critical depletion of cerebral extracellular glucose. ${ }^{1,2}$ This phenomenon may be further aggravated when availability of systemic glucose is limited, such as by intensive insulin therapy. ${ }^{24}$ Importantly, brain energy dysfunction and low CMD glucose is associated with worse outcome after TBI. ${ }^{2}$ Current therapeutic strategies for the management of secondary cerebral damage after TBI have mainly been focused on improving cerebral perfusion and controlling elevated $\mathrm{ICP} .{ }^{25}$ Growing evidence showing that brain energy dysfunction may be an important de- terminant of prognosis provides a robust rationale to test other therapeutic strategies aimed at improving neuroenergetics. ${ }^{26}$

\section{Hypertonic lactate to treat energy dysfunction after TBI}

Given the known deleterious role of hyperglycemia following brain injury, the administration of glucose-containing solutions to improve cerebral glucose cannot be recommended in this context and has generally been avoided, partly also because of the risk of increasing cerebral edema by the use of hypotonic solutions.

Lactate is an alternative energy substrate for the brain and can even act as preferential fuel, particularly in conditions of increased energy demand, such as those seen after injury. ${ }^{5,11,27}$ In vitro, lactate allows neural function to be maintained and prevents neuronal cell death upon hypoxia or glucose deprivation. ${ }^{28-30}$ Experimental blockade of lactate transport is associated with altered cognitive function in animals, reinforcing the notion that lactate may not only be an important substrate but also can serve as important signaling molecule for plasticity. ${ }^{31}$

Isotopic studies in patients with TBI appear to confirm that lactate can be used as an energy source by the injured brain. ${ }^{32}$ In healthy humans receiving HL, the contribution of exogenous systemic lactate to brain metabolism might increase sixfold up to $60 \% .^{33}$ Supplemental HL might therefore spare cerebral glucose ${ }^{34}$ and increase cerebral resistance to strenuous efforts ${ }^{19}$ and hypoglycemia. ${ }^{35} \mathrm{~A}$ large body of evidence suggests that HL exerts significant neuroprotection in several models of experimental brain injury, including TBI. ${ }^{12,13,36-38}$ In patients with TBI, HL might prevent secondary intracranial hypertension ${ }^{6}$ and is more effective than mannitol in controlling elevated ICP. ${ }^{7}$ Our group has recently shown that, in addition to reduce ICP, HL might further improve cerebral energetics in humans with TBI. ${ }^{8}$

\section{Improvement of cerebral glucose by hypertonic lactate seems dependent on baseline brain metabolic state}

The LPR reflects the metabolic state of the tissue, and an elevated LPR $>25$ is a marker of impaired cerebral oxidative metabolism suggesting elevated energy needs and/or compromised metabolic pathways. ${ }^{9}$ Elevated LPR $>25$ was also associated with neurological outcome in a large TBI cohort. ${ }^{10}$ In our study, more than half of patients had an LPR $>25$, consistent with previous studies. ${ }^{10}$ HL was associated with a significant and clinically

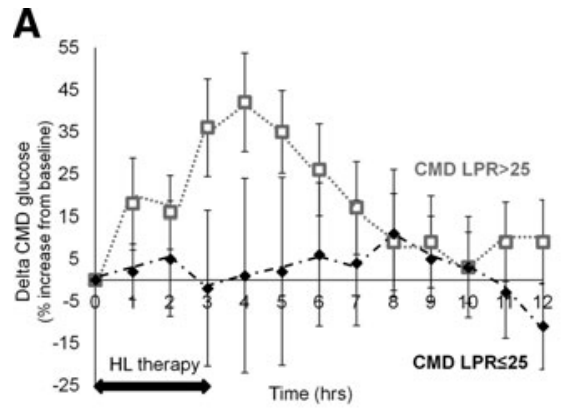

B

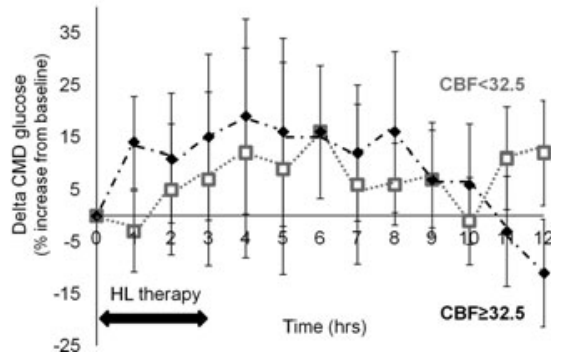

FIG. 2. Dynamic changes of cerebral extracellular glucose concentrations from baseline, during and following hypertonic lactate infusion (HL), according to patient subgroups, dichotomized by cerebral microdialysis (CMD) lactate/pyruvate ratio (LPR), or cerebral blood flow (CBF). The graph shows changes (expressed as the \% [delta] increase or decrease from baseline) of cerebral extracellular glucose over time following a 3-h intravenous HL, according to patient baseline LPR (A, dichotomized as normal [CMD LPR $\leq 25$; $n=11]$ vs. elevated [LPR $>25 ; n=13]$ ) and CBF (B, dichotomized as normal [CBF $\geq 32.5 \mathrm{~mL} / 100 \mathrm{~g} / \mathrm{min} ; n=14]$ vs. oligemic [CBF $<32.5 \mathrm{~mL} / 100 \mathrm{~g} / \mathrm{min} ; n=8])$. Data are expressed as mean \pm standard error of mean. 
Table 4. Changes of Cerebral Extracellular Glucose during Hypertonic lactate Infusion

\begin{tabular}{lccc}
\hline Condition & Coefficient \pm SE & 95\% CI & P value \\
\hline Cerebral LPR $>25(n=13)$ & $0.13 \pm 0.03$ & $0.08-0.19$ & $<0.0001$ \\
Cerebral LPR $\leq 25(n=11)$ & $0.04 \pm 0.04$ & $-0.05-0.13$ & 0.327 \\
$\mathrm{CBF}<32.5 \mathrm{~mL} / 100 \mathrm{~g} / \mathrm{min}(n=8)^{\mathrm{a}}$ & $0.13 \pm 0.04$ & $0.04-0.21$ & 0.003 \\
$\mathrm{CBF} \geq 32.5 \mathrm{~mL} / 100 \mathrm{~g} / \mathrm{min}(n=14)^{\mathrm{a}}$ & $0.09 \pm 0.03$ & $0.04-0.14$ & 0.001 \\
\hline
\end{tabular}

Changes are according to baseline CBF (dichotomized as oligemic $<32.5 \mathrm{~mL} / 100 \mathrm{~g} / \mathrm{min}$ vs. normal) and cerebral extracellular LPR (elevated $>25 \mathrm{vs}$. normal). $P$ values were calculated with mixed-effects multilevel regression model, to account for repeated measures across different patients over time. ${ }^{\mathrm{a}} \mathrm{PCT}$ data from two patients missing.

CBF, cerebral blood flow; CI, confidence interval; LPR, cerebral extracellular lactate/pyruvate ratio; SE, standard error.

relevant increase in cerebral glucose only in patients with baseline LPR $>25$, whereas no significant increase was observed when LPR was $<25$ at the start of intervention. This supports the notion that preferential lactate use with concomitant cerebral glucose sparing was correlated to patient brain metabolic state and was clearly apparent and clinically relevant ( $\sim 50 \%$ CMD glucose increase) in conditions where cerebral oxidative metabolism is impaired and tissue energy demand is increased. We have previously shown that CMD increase in pyruvate was also a function of baseline LPR. ${ }^{8}$ Altogether our findings suggest that CMD LPR can be used as a marker and potential end-point for therapeutic interventions aimed at improving neuroenergetics.

\section{Effect of hypertonic lactate in conditions of reduced $C B F$}

Whether HL might still be effective in conditions of reduced or impaired cerebral perfusion or oxygenation remains unclear. Of interest, Rosafio and colleagures recently found that hypoxia induces an overexpression of MCT4 in cultured astrocytes as well as enhanced lactate production, an effect that is viewed as a neuroprotective response. ${ }^{39}$ To answer this question, averaged values from right and left hemispheres were calculated, to quantify globally the extent of impaired CBF in each patient. We found that the effect of HL on cerebral glucose increase did not seem to be dependent on baseline CBF. However, average CBF was 29.6 (range 24.7-32) $\mathrm{mL} / 100 \mathrm{~g} / \mathrm{min}$, therefore well above the recognized definition of cerebral ischemia. Actually, some patients even displayed hyperemic CBF. Therefore, our data clearly support the finding that an elevated $\mathrm{CBF}$ does not seem to affect the metabolic response to HL infusion. Our study therefore lacks sufficient power to provide insights into the likely response of ischemic regions to hypertonic lactate. Additional studies in patients with ischemic/ hemorrhagic stroke are needed.

\section{Cerebral glucose-sparing effect of hypertonic lactate is independent of systemic glucose}

Supplemental systemic lactate may be metabolized into glucose via hepatic and renal GNG. Following TBI, increased systemic lactate metabolism and mobilization serves hepatic and renal GNG and can be an important source of glucose. ${ }^{21}$ This implies that increased CMD glucose following HL may be because of increased systemic glucose via induced GNG. Using a mixed-effects regression model, we found that systemic glucose did not increase significantly, which rather suggests a primary increase of cerebral lactate metabolism and supports the notion that lactate can indeed be used as a preferential energy substrate over glucose, as previously reported. ${ }^{34,40}$ Preferential lactate utilization will enable the "diversion" of glucose to become available for other important non-energetic modulatory and protective functions in the setting of TBI, for example, the pentose phosphate pathway. ${ }^{41}$

\section{Study limitations}

Cerebral metabolism was assessed regionally with CMD, but we did not measure global cerebral metabolism with positron emission tomography. This is a limitation that is compounded by the fact that CMD technique is readily available in the critical care setting and is part of standard patient care. Similarly, CBF was measured with PCT, which explores CBF partially: only anterior and middle cerebral vascular territories were assessed and changes in posterior cerebral vascular territories may be missed. PCT has been previously validated by our group in patients with severe $\mathrm{TBI}^{14-16}$ with good accuracy for the measurement of CBF when compared with gold-standard techniques, such as xenon $\mathrm{CT} .{ }^{42}$ $\mathrm{CBF}$ is dynamic especially within the first $72 \mathrm{~h}$ after injury, so this could affect the overall findings and must be recognized as a potential limitation of our study. Our definition of low CBF deserves further discussion. Oligemic $\mathrm{CBF}<32.5 \mathrm{~mL} / 100 \mathrm{~g} / \mathrm{min}$ was about 2 standard deviations below normal CBF. Although this threshold could be considered as only marginally low and above the classical ischemic range $(18-22 \mathrm{~mL} / 100 \mathrm{~g} / \mathrm{min})$, it has both robust clinical ${ }^{16}$ and prognostic ${ }^{15}$ value. In addition, partly because of the modern setting of protocol-driven management, true ischemic states are no longer common after TBI in the neurocritical care phase. ${ }^{43}$ In our study, the "pro-energetic" effect of HL did not seem to be reduced in conditions of limited CBF. However, we did not test HL in conditions of overt ischemia. The inability to address this essential question is a serious limitation, but we believe our findings at least provide a reasonable rationale to further test the value of HL in patients with unequivocally ischemic brain regions.

\section{Conclusion}

Our findings suggest that improvement of brain energetics during infusion of HL is predominantly dependent on baseline cerebral metabolic state and support the concept that an elevated LPR $>25$ after TBI is associated with a greater likelihood of brain glucose increase as lactate is preferentially used as an energy substrate. In this setting, LPR can be used as a diagnostic indication for HL. This study also suggests that because HL effect on metabolism in tissue with elevated LPR seems to be independent of CBF, perhaps it would work well in tissue with non-ischemic LPR elevation and could also be tested in other acute cerebral conditions where CBF was more profoundly limited. 


\section{Acknowledgments}

This work was supported by research grants from the Swiss National Science Foundation (to MO), the Novartis Foundation for Biomedical Research (to MO), and the Société Française d'Anesthésie et Réanimation (SFAR, to HQ).

\section{Author Disclosure Statement}

No conflicting financial interests exist.

\section{References}

1. Glenn, T.C., Kelly, D.F., Boscardin, W.J., McArthur, D.L., Vespa, P., Oertel, M., Hovda, D.A., Bergsneider, M., Hillered, L., and Martin, N.A. (2003). Energy dysfunction as a predictor of outcome after moderate or severe head injury: indices of oxygen, glucose, and lactate metabolism. J. Cereb. Blood Flow Metab. 23, 1239-1250.

2. Vespa, P.M., McArthur, D., O'Phelan, K., Glenn, T., Etchepare, M., Kelly, D., Bergsneider, M., Martin, N.A., and Hovda, D.A. (2003). Persistently low extracellular glucose correlates with poor outcome 6 months after human traumatic brain injury despite a lack of increased lactate: a microdialysis study. J. Cereb. Blood Flow Metab. 23, 865877

3. Mergenthaler, P., Lindauer, U., Dienel, G.A., and Meisel, A. (2013). Sugar for the brain: the role of glucose in physiological and pathological brain function. Trends Neurosci. 36, 587-597.

4. Pellerin, L., and Magistretti, P.J. (2012). Sweet sixteen for ANLS. J. Cereb. Blood Flow Metab. 32, 1152-1166.

5. Barros, L.F. (2013). Metabolic signaling by lactate in the brain. Trends Neurosci. 36, 396-404.

6. Ichai, C., Payen, J.F., Orban, J.C., Quintard, H., Roth, H., Legrand, R. Francony, G., and Leverve, X.M. (2013). Half-molar sodium lactate infusion to prevent intracranial hypertensive episodes in severe traumatic brain injured patients: a randomized controlled trial. Intensive Care Med. 39, 1413-1422.

7. Ichai, C., Armando, G., Orban, J.C., Berthier, F., Rami, L., Samat-Long, C., Grimaud, D., and Leverve, X. (2009). Sodium lactate versus mannitol in the treatment of intracranial hypertensive episodes in severe traumatic brain-injured patients. Intensive Care Med. 35, 471-479.

8. Bouzat, P., Sala, N., Suys, T., Zerlauth, J.B., Marques-Vidal, P., Feihl, F., Bloch, J., Messerer, M., Levivier, M., Meuli, R., Magistretti, P.J., and Oddo, M. (2014). Cerebral metabolic effects of exogenous lactate supplementation on the injured human brain. Intensive Care Med. 40, 412-421.

9. Reinstrup, P., Stahl, N., Mellergard, P., Uski, T., Ungerstedt, U., and Nordstrom, C.H. (2000). Intracerebral microdialysis in clinical practice: baseline values for chemical markers during wakefulness, anesthesia, and neurosurgery. Neurosurgery 47, 701-709; discussion 709-710.

10. Timofeev, I., Carpenter, K.L., Nortje, J., Al-Rawi, P.G., O’Connell, M.T., Czosnyka, M., Smielewski, P., Pickard, J.D., Menon, D.K., Kirkpatrick, P.J., Gupta, A.K., and Hutchinson, P.J. (2011). Cerebral extracellular chemistry and outcome following traumatic brain injury: a microdialysis study of 223 patients. Brain 134, 484-494.

11. Bouzat, P., and Oddo, M. (2014). Lactate and the injured brain: friend or foe? Curr. Opinion Crit. Care 20, 133-140.

12. Berthet, C., Castillo, X., Magistretti, P.J., and Hirt, L. (2012). New evidence of neuroprotection by lactate after transient focal cerebral ischaemia: extended benefit after intracerebroventricular injection and efficacy of intravenous administration. Cerebrovasc. Dis. 34, 329-335.

13. Berthet, C., Lei, H., Thevenet, J., Gruetter, R., Magistretti, P.J., and Hirt, L. (2009). Neuroprotective role of lactate after cerebral ischemia. J. Cereb. Blood Flow Metab. 29, 1780-1789.

14. Sala, N., Suys, T., Zerlauth, J.B., Bouzat, P., Messerer, M., Bloch, J., Levivier, M., Magistretti, P.J., Meuli, R., and Oddo, M. (2013). Cerebral extracellular lactate increase is predominantly nonischemic in patients with severe traumatic brain injury. J. Cereb. Blood Flow Metab. 33, 1815-1822.

15. Wintermark, M., van Melle, G., Schnyder, P., Revelly, J.P., Porchet, F., Regli, L., Meuli, R., Maeder, P., and Chiolero, R. (2004). Admission perfusion CT: prognostic value in patients with severe head trauma. Radiology 232, 211-220.
16. Bouzat, P., Marques-Vidal, P., Zerlauth, J.B., Sala, N., Suys, T., Schoettker, P., Bloch, J., Daniel, R.T., Levivier, M., Meuli, R., and Oddo, M. (2015). Accuracy of brain multimodal monitoring to detect cerebral hypoperfusion after traumatic brain injury*. Crit. Care Med. 43, 445-452.

17. Dalsgaard, M.K., Quistorff, B., Danielsen, E.R., Selmer, C., Vogelsang, T., and Secher, N.H. (2004). A reduced cerebral metabolic ratio in exercise reflects metabolism and not accumulation of lactate within the human brain. J. Physiology 554, 571-578.

18. Quistorff, B., Secher, N.H., and Van Lieshout, J.J. (2008). Lactate fuels the human brain during exercise. F.A.S.E.B. J. 22, 3443-3449.

19. van Hall, G., Stromstad, M., Rasmussen, P., Jans, O., Zaar, M., Gam, C., Quistorff, B., Secher, N.H., and Nielsen, H.B. (2009). Blood lactate is an important energy source for the human brain. J. Cereb. Blood Flow Metab. 29, 1121-1129.

20. Levy, B., Gibot, S., Franck, P., Cravoisy, A., and Bollaert, P.E. (2005). Relation between muscle $\mathrm{Na}+\mathrm{K}+$ ATPase activity and raised lactate concentrations in septic shock: a prospective study. Lancet 365, 871875 .

21. Glenn, T.C., Martin, N.A., McArthur, D.L., Hovda, D., Vespa, P.M.M., Horning, M.A., Johnson, M.L., and Brooks, G.A. (2015). Endogenous nutritive support following traumatic brain injury: peripheral lactate production for glucose supply via gluconeogenesis. J. Neurotrauma 32, 820-832.

22. Simpson, I.A., Carruthers, A., and Vannucci, S.J. (2007). Supply and demand in cerebral energy metabolism: the role of nutrient transporters. J. Cereb. Blood Flow M. 27, 1766-1791.

23. Bergsneider, M., Hovda, D.A., Shalmon, E., Kelly, D.F., Vespa, P.M., Martin, N.A., Phelps, M.E., McArthur, D.L., Caron, M.J., Kraus, J.F., and Becker, D.P. (1997). Cerebral hyperglycolysis following severe traumatic brain injury in humans: a positron emission tomography study. J. Neurosurgery 86, 241-251.

24. Vespa, P., McArthur, D.L., Stein, N., Huang, S.C., Shao, W., Filippou, M., Etchepare, M., Glenn, T., and Hovda, D.A. (2012). Tight glycemic control increases metabolic distress in traumatic brain injury: a randomized controlled within-subjects trial. Crit. Care Med. 40, 19231929.

25. Maas, A.I., Stocchetti, N., and Bullock, R. (2008). Moderate and severe traumatic brain injury in adults. Lancet. Neurology 7, 728-741.

26. Bouzat, P., Sala, N., Payen, J.F., and Oddo, M. (2013). Beyond intracranial pressure: optimization of cerebral blood flow, oxygen, and substrate delivery after traumatic brain injury. Ann. Intensive Care 3, 23.

27. Patet, C., Quintard, H., Suys, T., Bloch, J., Daniel, R., Pellerin, L., Magistretti, P., and Oddo, M. (2015). Neuroenergetic response to prolonged cerebral glucose depletion after severe brain injury and the role of lactate. J. Neurotrauma 32, 1560-1566.

28. Bouzier-Sore, A.K., Voisin, P., Canioni, P., Magistretti, P.J., and Pellerin, L. (2003). Lactate is a preferential oxidative energy substrate over glucose for neurons in culture. J. Cereb. Blood Flow Metab. 23, 1298-1306.

29. Cater, H.L., Benham, C.D., and Sundstrom, L.E. (2001). Neuroprotective role of monocarboxylate transport during glucose deprivation in slice cultures of rat hippocampus. J. Physiology 531, 459-466.

30. Schurr, A., Payne, R.S., Miller, J.J., and Rigor, B.M. (1997). Brain lactate is an obligatory aerobic energy substrate for functional recovery after hypoxia: further in vitro validation. J. Neurochemistry 69 , 423-426.

31. Yang, J., Ruchti, E., Petit, J.M., Jourdain, P., Grenningloh, G., Allaman, I., and Magistretti, P.J. (2014). Lactate promotes plasticity gene expression by potentiating NMDA signaling in neurons. Proc. Natl. Acad. Sci. U. S. A. 111, 12228-12233.

32. Gallagher, C.N., Carpenter, K.L., Grice, P., Howe, D.J., Mason, A., Timofeev, I., Menon, D.K., Kirkpatrick, P.J., Pickard, J.D., Sutherland, G.R., and Hutchinson, P.J. (2009). The human brain utilizes lactate via the tricarboxylic acid cycle: a 13C-labelled microdialysis and high-resolution nuclear magnetic resonance study. Brain 132, 2839-2849.

33. Boumezbeur, F., Petersen, K.F., Cline, G.W., Mason, G.F., Behar, K.L., Shulman, G.I., and Rothman, D.L. (2010). The contribution of blood lactate to brain energy metabolism in humans measured by dynamic 13C nuclear magnetic resonance spectroscopy. J. Neuroscience 30, 13983-13991.

34. Smith, D., Pernet, A., Hallett, W.A., Bingham, E., Marsden, P.K., and Amiel, S.A. (2003). Lactate: a preferred fuel for human brain metabolism in vivo. J. Cereb. Blood Flow Metab. 23, 658-664. 
35. Maran, A., Crepaldi, C., Trupiani, S., Lucca, T., Jori, E., Macdonald, I.A., Tiengo, A., Avogaro, A., and Del Prato, S. (2000). Brain function rescue effect of lactate following hypoglycaemia is not an adaptation process in both normal and type I diabetic subjects. Diabetologia 43, 733-741.

36. Herzog, R.I., Jiang, L., Herman, P., Zhao, C., Sanganahalli, B.G., Mason, G.F., Hyder, F., Rothman, D.L., Sherwin, R.S., and Behar, K.L. (2013). Lactate preserves neuronal metabolism and function following antecedent recurrent hypoglycemia. J. Clin. Invest. 123, 1988-1998.

37. Rice, A.C., Zsoldos, R., Chen, T., Wilson, M.S., Alessandri, B., Hamm, R.J., and Bullock, M.R. (2002). Lactate administration attenuates cognitive deficits following traumatic brain injury. Brain Res. 928, 156-159.

38. Ros, J., Pecinska, N., Alessandri, B., Landolt, H., and Fillenz, M. (2001). Lactate reduces glutamate-induced neurotoxicity in rat cortex. J. Neurosci. Res. 66, 790-794.

39. Rosafio, K., and Pellerin, L. (2014). Oxygen tension controls the expression of the monocarboxylate transporter MCT4 in cultured mouse cortical astrocytes via a hypoxia-inducible factor-1alpha-mediated transcriptional regulation. Glia 62, 477-490.

40. Sotelo-Hitschfeld, T., Fernandez-Moncada, I., and Barros, L.F. (2012). Acute feedback control of astrocytic glycolysis by lactate. Glia 60, 674-680.

41. Jalloh, I., Carpenter, K.L., Grice, P., Howe, D.J., Mason, A., Gallagher, C.N., Helmy, A., Murphy, M.P., Menon, D.K., Carpenter, T.A.,
Pickard, J.D., and Hutchinson, P.J. (2015). Glycolysis and the pentose phosphate pathway after human traumatic brain injury: microdialysis studies using 1,2-(13)C2 glucose. J. Cereb. Blood Flow Metab. 35, 111-120.

42. Wintermark, M., Thiran, J.P., Maeder, P., Schnyder, P., and Meuli, R. (2001). Simultaneous measurement of regional cerebral blood flow by perfusion CT and stable xenon CT: a validation study. A.J.N.R. 22, 905-914.

43. Vespa, P., Bergsneider, M., Hattori, N., Wu, H.M., Huang, S.C., Martin, N.A., Glenn, T.C., McArthur, D.L., and Hovda, D.A. (2005). Metabolic crisis without brain ischemia is common after traumatic brain injury: a combined microdialysis and positron emission tomography study. J. Cereb. Blood Flow Metabolism 25, 763-774.

Address correspondence to:

Mauro Oddo, MD

Department of Intensive Care Medicine CHUV-University Hospital

Rue du Bugnon 46, BH 08.623

CH-1011 Lausanne, Switzerland

E-mail: mauro.oddo@chuv.ch 